\title{
FIXED POINT THEOREMS FOR SET-VALUED $Y$-CONTRACTIONS
}

\author{
IOAN A. RUS, ADRIAN PETRUŞEL and GABRIELA PETRUŞEL \\ Department of Applied Mathematics, Babeş-Bolyai University Cluj-Napoca \\ Kogălniceanu 1, 400084 Cluj-Napoca, Romania \\ E-mails:iarus@math.ubbcluj.ro,petrusel@math.ubbcluj.ro,gabip@math.ubbcluj.ro
}

\begin{abstract}
The purpose of this paper is to present several fixed point theorems for the socalled set-valued $Y$-contractions. Set-valued $Y$-contractions in ordered metric spaces, set-valued graphic contractions, set-valued contractions outside a bounded set and set-valued operators on a metric space with cyclic representations are considered.
\end{abstract}

\section{Introduction}

1.1. Basic notions and notation. Throughout this paper, the standard notation and terminology in nonlinear analysis are used. For the convenience of the reader we recall some of them.

Let $(X, d)$ be a metric space and $f: X \rightarrow X$ an operator. By Fix $f:=\{x \in X:$ $x=f(x)\}$ we will denote the fixed point set of the operator $f$ and by $\tilde{B}\left(x_{0}, r\right)$ the closed ball centered in $x_{0} \in X$ with radius $r>0$.

We will also use the following symbols:

$$
\begin{array}{ll}
P(X):=\{Y \subset X: Y \text { is nonempty }\}, & P_{\mathrm{cl}}(X):=\{Y \in P(X): Y \text { is closed }\}, \\
P_{\mathrm{cp}}(X):=\{Y \in P(X): Y \text { is compact }\}, & P_{\mathrm{b}}(X):=\{Y \in P(X): Y \text { is bounded }\} .
\end{array}
$$

In normed spaces, $P_{\mathrm{cv}}(X):=\{Y \in P(X): Y$ is convex $\}$.

Let $A$ and $B$ be nonempty subsets of the metric space $(X, d)$. The gap between these sets is

$$
D(A, B)=\inf \{d(a, b): a \in A, b \in B\} .
$$

In particular, $D\left(x_{0}, B\right)=D\left(\left\{x_{0}\right\}, B\right.$ ) (where $\left.x_{0} \in X\right)$ is called the distance from the point $x_{0}$ to the set $B$.

2000 Mathematics Subject Classification: 47H10, 54H25, 47H06.

Key words and phrases: fixed point, strict fixed point, selection, multi-valued operator, multivalued weakly Picard operator, contraction outside a bounded set, cyclic representation.

The paper is in final form and no version of it will be published elsewhere. 
The Pompeiu-Hausdorff generalized distance between the nonempty closed subsets $A$ and $B$ of the metric space $(X, d)$ is defined by the formula

$$
H(A, B):=\max \left\{\sup _{a \in A} \inf _{b \in B} d(a, b), \sup _{b \in B} \inf _{a \in A} d(a, b)\right\} .
$$

If $A$ and $B$ are nonempty and bounded subsets of the metric space $(X, d)$, then one defines

$$
\delta(A, B):=\sup \{d(a, b): a \in A, b \in B\} .
$$

The symbol $T: X \multimap Y$ means $T: X \rightarrow P(Y)$, i.e., $T$ is a set-valued operator from $X$ to $Y$. We will denote by $\mathbb{M}^{0}(X, Y)$ the set of all set-valued operators $T: X \multimap Y$. If $X=Y$ then $\mathbb{M}^{0}(Y):=\mathbb{M}^{0}(Y, Y)$. For $K \in P(X), T(K):=\bigcup_{x \in K} T(x)$ will denote the image of the set $K$, while $\operatorname{Graf}(T):=\{(x, y) \in X \times Y: y \in T(x)\}$ is the graph of $T$. Recall that the set-valued operator is called closed if $\operatorname{Graf}(T)$ is closed in $X \times Y$. If $X$ and $Y$ are Hausdorff topological spaces then $T^{+}(U):=\{x \in X: T(x) \subset U\}$, for $U \in P(Y)$. The set-valued operator $T: X \rightarrow P(Y)$ is said to be upper semi-continuous on $X$ (briefly u.s.c.) if and only if $T^{+}(U)$ is open in $X$ for each open subset $U$ of $Y$.

For $T: X \rightarrow P(X)$ the set of all nonempty invariant subsets of $X$ will be denoted by $I(T)$, i.e. $I(T):=\{Y \in P(X): T(Y) \subset Y\}$. Also $\operatorname{Fix}(T):=\{x \in X: x \in T(x)\}$ denotes the fixed point set of the set-valued operator $T$, while $\operatorname{SFix}(T):=\{x \in X:\{x\}=T(x)\}$ is the strict fixed point set of $T$.

If $(X, d)$ is a metric space, recall that $T: X \rightarrow P_{\mathrm{cl}}(X)$ is called a set-valued $a$-contraction if $a \in] 0,1\left[\right.$ and $H\left(T\left(x_{1}\right), T\left(x_{2}\right)\right) \leq a \cdot d\left(x_{1}, x_{2}\right)$, for each $x_{1}, x_{2} \in X$. In the same setting, an operator $T: X \rightarrow P_{\mathrm{cl}}(X)$ is a multivalued weakly Picard operator (briefly $M W P$ operator) (see [25]) if for each $x \in X$ and each $y \in T(x)$ there exists a sequence $\left(x_{n}\right)_{n \in \mathbb{N}}$ in $X$ such that:

(i) $x_{0}=x, x_{1}=y$;

(ii) $x_{n+1} \in T\left(x_{n}\right)$ for all $n \in \mathbb{N}$;

(iii) the sequence $\left(x_{n}\right)_{n \in \mathbb{N}}$ is convergent and its limit is a fixed point of $T$.

A sequence $\left(x_{n}\right)_{n \in \mathbb{N}}$ in $X$ satisfying the condition (ii) from the previous definition is called the sequence of successive approximations of $T$ starting from $x_{0} \in X$.

(Here $\mathbb{N}$ denotes the set of all nonnegative integers. The set of all positive integers will be denoted by $\mathbb{N}^{*}$.)

Let $X$ be a nonempty set. By definition (see [20]), the triple $\left(X, S(X), M^{0}\right)$ is a fixed point structure (briefly f.p.s.) if:

(i) $S(X) \subset P(X), S(X) \neq \emptyset$;

(ii) $M^{0}: P(X) \multimap \bigcup_{Y \in P(X)} \mathbb{M}^{0}(Y)$, with $Y \multimap M^{0}(Y) \subset \mathbb{M}^{0}(Y)$ is a mapping such that if $Z \subset Y, Z \neq \emptyset$ then $M^{0}(Z) \supset\left\{T_{\left.\right|_{Z}}: T \in M^{0}(Y), Z \in I(T)\right\}$;

(iii) every $Y \in S(X)$ has the fixed point property with respect to $M^{0}(Y)$.

Let $X$ be a nonempty set. Define $s(X):=\left\{\left(x_{n}\right)_{n \in \mathbb{N}}: x_{n} \in X, n \in \mathbb{N}\right\}$.

Let $c(X) \subset s(X)$ be a subset of $s(X)$ and Lim $: c(X) \rightarrow X$ an operator. By definition the triple $(X, c(X), \mathrm{Lim})$ is called an $L$-space (Fréchet [4]) if the following conditions are 
satisfied:

(i) If $x_{n}=x$ for each $n \in \mathbb{N}$, then $\left(x_{n}\right)_{n \in \mathbb{N}} \in c(X)$ and $\operatorname{Lim}\left(x_{n}\right)_{n \in \mathbb{N}}=x$.

(ii) If $\left(x_{n}\right)_{n \in \mathbb{N}} \in c(X)$ and $\operatorname{Lim}\left(x_{n}\right)_{n \in \mathbb{N}}=x$, then for all subsequences, $\left(x_{n_{i}}\right)_{i \in \mathbb{N}}$, of $\left(x_{n}\right)_{n \in \mathbb{N}}$ we have $\left(x_{n_{i}}\right)_{i \in \mathbb{N}} \in c(X)$ and $\operatorname{Lim}\left(x_{n_{i}}\right)_{i \in \mathbb{N}}=x$.

By definition an element of $c(X)$ is a convergent sequence and $x:=\operatorname{Lim}\left(x_{n}\right)_{n \in \mathbb{N}}$ is the limit of this sequence and we write $x_{n} \rightarrow x$ as $n \rightarrow \infty$.

In what follow we denote an L-space by $(X, \rightarrow)$. Hausdorff topological spaces, metric spaces, generalized metric spaces $\left(d(x, y) \in \mathbb{R}_{+}^{m}\right.$, or $d(x, y) \in \mathbb{R}_{+} \cup\{+\infty\}$, or $d(x, y) \in K$, $K$ a cone in an ordered Banach space, or $d(x, y) \in E, E$ an ordered linear space with a notion of linear convergence, etc.), gauge spaces, 2-metric spaces, D-R-spaces, probabilistic metric spaces, syntopogenous spaces, are examples of L-spaces.

1.2. The aim of the paper. The purpose of this work is to consider the following open question of I. A. Rus [21]:

I. Let $(X, d)$ be a metric space and $Y \subseteq X \times X$. The operator $T: X \rightarrow P_{\mathrm{cl}}(X)$ is called $a$ set-valued $(Y, a)$-contraction if

$$
a \in] 0,1\left[\text { and } H\left(T\left(x_{1}\right), T\left(x_{2}\right)\right) \leq a \cdot d\left(x_{1}, x_{2}\right) \text { for each }\left(x_{1}, x_{2}\right) \in Y .\right.
$$

Construct a fixed point theory for set-valued $(Y, a)$-contractions.

Of course, in the previous definition, one can imagine generalized type conditions for $T$, such as

there exists $\varphi: \mathbb{R}_{+}^{5} \rightarrow \mathbb{R}_{+}$such that

$$
\begin{array}{r}
H\left(T\left(x_{1}\right), T\left(x_{2}\right)\right) \leq \varphi\left(d\left(x_{1}, x_{2}\right), D\left(x_{1}, T\left(x_{1}\right)\right), D\left(x_{2}, T\left(x_{2}\right)\right), D\left(x_{1}, T\left(x_{2}\right)\right), D\left(x_{2}, T\left(x_{1}\right)\right)\right) \\
\text { for each }\left(x_{1}, x_{2}\right) \in Y . \quad(1.2)
\end{array}
$$

In connection with the above problem one can consider

II. Let $(X, d)$ be a metric space and $Y \subseteq X \times X$. The operator $T: X \rightarrow P_{\mathrm{b}}(X)$ is called $a$ set-valued $(Y, a)$ - $\delta$-contraction if

$$
a \in] 0,1\left[\text { and } \delta\left(T\left(x_{1}\right), T\left(x_{2}\right)\right) \leq a \cdot d\left(x_{1}, x_{2}\right) \text { for each }\left(x_{1}, x_{2}\right) \in Y .\right.
$$

Construct a fixed point theory for set-valued $(Y, a)-\delta$-contractions.

III. Let $(X, d)$ be a metric space and $Y \subseteq X \times X$. The operator $T: X \rightarrow P_{\mathrm{cl}}(X)$ is called a set-valued $(Y, a)$-Gap-contraction if

$$
a \in] 0,1\left[\text { and } D\left(T\left(x_{1}\right), T\left(x_{2}\right)\right) \leq a \cdot d\left(x_{1}, x_{2}\right) \text { for each }\left(x_{1}, x_{2}\right) \in Y .\right.
$$

Construct a fixed point theory for set-valued $(Y, a)$-Gap-contractions.

Obviously, as in case I, generalized type conditions on $T$ can be also considered.

The following examples are in connection with the above problems.

EXAmPLE 1.1. Let $(X, d)$ be a metric space and $Y:=X \times X$. Then a set-valued $(Y, a)$ contraction is a set-valued $a$-contraction ([1], [2], [3], [6], [8], [11], [16], [21], [26], etc.).

Example 1.2. Let $(X, d)$ be a metric space, $T: X \rightarrow P_{\mathrm{cl}}(X)$ and $Y:=\operatorname{Graf}(T)$. Then a set-valued $(Y, a)$-contraction is a set-valued $a$-graphic contraction ([17], [18]). 
EXAmple 1.3. Let $(X, d)$ be a metric space, $x_{0} \in X$ and $r>0$. Consider $Y:=\tilde{B}\left(x_{0}, r\right) \times$ $\tilde{B}\left(x_{0}, r\right)$. Then for $T: X \rightarrow P_{\mathrm{cl}}(X)$ the set-valued $(Y, a)$-contraction condition means that the following Frigon-Granas type condition (see [5], [12]) holds:

there is $a \in] 0,1\left[\operatorname{such}\right.$ that $H(T(x), T(y)) \leq a \cdot d(x, y)$ for all $x, y \in \tilde{B}\left(x_{0}, r\right)$.

ExAmple 1.4. Let $(X, d)$ be a metric space, $Z \in P_{\mathrm{b}}(X)$ and $Y:=(X \backslash Z) \times(X \backslash Z)$. Then a set-valued $(Y, a)$-contraction is a set-valued $a$-contraction outside a bounded subset, see [21], [19].

Example 1.5. Let $(X, d, \leq)$ be an ordered metric space. Consider $Y:=\{(x, y) \in X \times X$ : $x \leq y$ or $y \leq x\}$. Then $T: X \rightarrow P_{\mathrm{cl}}(X)$ is a set-valued $(Y, a)$-contraction if (see also $[15],[13])$ if

$$
a \in] 0,1[\text { and } H(T(x), T(y)) \leq a \cdot d(x, y) \text {, for all } x, y \in X, x \leq y .
$$

Example 1.6. Let $(X, d)$ be a metric space, $T: X \rightarrow P_{\mathrm{cl}}(X)$ and $A_{i} \in P(X)$, $i \in\{1,2, \ldots, m\}$. Suppose that $X=\bigcup_{i=1}^{m} A_{i}$ is a cyclic representation with respect to the set-valued operator $T$, i.e. $T\left(A_{i}\right) \subset A_{i+1}, i \in\{1,2, \ldots, m\}$ (where $A_{m+1}=A_{1}$ ).

Consider $Y:=\bigcup_{i=1}^{m}\left(A_{i} \times A_{i+1}\right)$. Then $T$ is a set-valued $(Y, a)$-contraction $([9],[23])$ if $a \in] 0,1[$ and $H(T(x), T(y)) \leq a \cdot d(x, y)$

for all $x \in A_{i}$ and all $y \in A_{i+1}, i \in\{1,2, \ldots, m\}$.

2. Set-valued $(Y, a)$-contractions in ordered metric spaces. Let $(X, \leq)$ be a partially ordered set. Let $X_{\leq}:=\{(x, y) \in X \times X: x \leq y$ or $y \leq x\}$. Also, if $x, y \in X$ with $x \leq y$, then by $[x, y] \leq$ we will denote the ordered segment joining $x$ and $y$, i.e. $[x, y]_{\leq}:=\{z \in X: x \leq z \leq y\}$.

Definition 2.1. Let $X$ be a nonempty set. Then, by definition $(X, d, \leq)$ is an ordered metric space if and only if:

(i) $(X, d)$ is a metric space;

(ii) $(X, \leq)$ is a partially ordered set;

(iii) $\left(x_{n}\right)_{n \in \mathbb{N}} \rightarrow x,\left(y_{n}\right)_{n \in \mathbb{N}} \rightarrow y$ and $x_{n} \leq y_{n}$ for each $n \in \mathbb{N} \Rightarrow x \leq y$.

The main result of this section is

TheOREM 2.2. Let $(X, d, \leq)$ be an ordered complete metric space and $T: X \rightarrow P_{\mathrm{cl}}(X)$ a set-valued operator. Suppose that the following assertions hold:

(i) there exists $x_{0} \in X$ such that if $y \in T\left(x_{0}\right)$ then $\left(x_{0}, y\right) \in X_{\leq}$;

(ii) for each $x, y \in X$, with $(x, y) \notin X_{\leq}$, there exists $c(x, y) \in X$ such that $(x, c(x, y))$ and $(y, c(x, y))$ belong to $X_{\leq}$;

(iii) $(x, y) \in X_{\leq}$implies $\left(u \in T(x)\right.$ and $v \in T(y)$ then $\left.(u, v) \in X_{\leq}\right)$;

(iv) $T$ is a closed set-valued operator;

(v) $T$ is a set-valued $\left(X_{\leq}, a\right)$-contraction. 
Then for each $x \in X$ there is a sequence $\left(x_{n}\right)_{n \in \mathbb{N}}$ of successive approximations of $T$ starting from $x$ that converges to a fixed point of $T$.

Proof. Let $q>1$ and $x_{1} \in T\left(x_{0}\right)$ be arbitrary. Then there exists $x_{2} \in T\left(x_{1}\right)$ such that $d\left(x_{1}, x_{2}\right) \leq q \cdot H\left(T\left(x_{0}\right), T\left(x_{1}\right)\right)$. From (i) we have $\left(x_{0}, x_{1}\right) \in X_{\leq}$. Using (v) we obtain $H\left(T\left(x_{0}\right), T\left(x_{1}\right)\right) \leq a \cdot d\left(x_{0}, x_{1}\right)$. As a consequence, $d\left(x_{1}, x_{2}\right) \leq q \cdot a \cdot d\left(x_{0}, x_{1}\right)$. Taking (iii) into account we get $\left(x_{1}, x_{2}\right) \in X_{\leq}$. Inductively we can construct a sequence $\left(x_{n}\right)_{n \in \mathbb{N}}$ having the following two properties:

(a) $x_{n+1} \in T\left(x_{n}\right)$ for $n \in \mathbb{N}$;

(b) $d\left(x_{n+1}, x_{n}\right) \leq q \cdot a \cdot d\left(x_{n}, x_{n-1}\right)$ for $n \in \mathbb{N}^{*}$.

If we choose $q \in] 1, a^{-1}$ [ then, by standard methods, we obtain the convergence of the sequence $\left(x_{n}\right)_{n \in \mathbb{N}}$ to some $x^{*} \in X$. From (iv) it follows that $x^{*} \in F_{T}$. Hence $\operatorname{Fix}(T) \neq \emptyset$.

We will prove now that for each $x \in X$ and each $y \in T(x)$ there exists a sequence $\left(z_{n}\right)_{n \in \mathbb{N}}$ of successive approximations for $T$ starting from $(x, y) \in \operatorname{Graf}(T)$ such that $\left(z_{n}\right)$ converges to a fixed point of $T$.

Case A. Let $z_{0} \in X$ such that $\left(x_{0}, z_{0}\right) \in X_{\leq}$. Then there exists $z_{1} \in T\left(z_{0}\right)$ such that $d\left(x_{1}, z_{1}\right) \leq q \cdot H\left(T\left(x_{0}\right), T\left(z_{0}\right)\right) \leq q \cdot a \cdot d\left(x_{0}, z_{0}\right)$. Since $\left(x_{1}, z_{1}\right) \in X_{\leq}$we can continue this procedure and obtain a sequence $\left(z_{n}\right)_{n \in \mathbb{N}}$ such that $z_{n+1} \in T\left(z_{n}\right)$ for $n \in \mathbb{N}$ and $d\left(x_{n+1}, z_{n+1}\right) \leq q \cdot a \cdot d\left(x_{n}, z_{n}\right)$. Choosing $\left.q \in\right] 1, a^{-1}\left[\right.$ we deduce that $\left(z_{n}\right) \rightarrow x^{*} \in T\left(x^{*}\right)$.

Case B. Let $z_{0} \in X$ such that $\left(x_{0}, z_{0}\right) \notin X_{\leq}$. Then, from (ii) there exists $c\left(x_{0}, z_{0}\right) \in X$ such that

$$
\left(x_{0}, c\left(x_{0}, z_{0}\right)\right) \in X_{\leq}
$$

and

$$
\left(z_{0}, c\left(x_{0}, z_{0}\right)\right) \in X_{\leq}
$$

From (1), using the method from Case A, we can construct a sequence of successive approximations for $T$ starting from $c\left(x_{0}, z_{0}\right)$ which converges to $x^{*}$. From $(2)$ and the above conclusion, we deduce in the same manner as in Case A that there exists a sequence $\left(z_{n}\right)$ of successive approximations for $T$ starting from $z_{0}$ such that $\left(z_{n}\right) \rightarrow x^{*}$. This finishes the proof.

REMARK 2.3. It is an open question whether $T$ satisfying the hypotheses of Theorem 2.2 is a MWP operator.

3. Set-valued graphic contractions. Let $(X, d)$ be a complete metric space and $T: X \rightarrow P_{b}(X)$ be a set-valued operator. If there exist $a, b, c \in \mathbb{R}_{+}$with $a+b+c<1$ such that

$$
\delta(T(x), T(y)) \leq a \cdot d(x, y)+b \cdot \delta(x, T(x))+c \cdot \delta(y, T(y)) \text { for each } x \in X \text { and } y \in T(x),
$$

then the problem is to study when $\operatorname{Fix}(T)=\operatorname{SFix}(T) \neq \emptyset$.

In connection with the above problem we have:

Example 3.1. Let $(X, d)$ be a metric space and $X=X_{1} \cup \ldots \cup X_{m}$ be a partition of $X$. For each $i \in\{1,2, \ldots, m\}$, let $x_{i} \in X_{i}$ be given elements. Consider $T: X \rightarrow P_{b}(X)$ 
defined by $T(x):=\left\{x_{i}\right\}$, if $x \in X_{i}$. Then $T$ satisfies the above condition and $\operatorname{Fix}(T)=$ $\operatorname{SFix}(T)=\left\{x_{1}, \ldots, x_{m}\right\}$.

The first result of this section is the following strict fixed point theorem for a set-valued operator satisfying to a certain contractive type condition on its graph.

Theorem 3.2. Let $(X, d)$ be a complete metric space and $T: X \rightarrow P_{b}(X)$ be a closed set-valued operator. Suppose that

there exist $a, b, c \in \mathbb{R}_{+}$with $a+b+c<1$ such that

$$
\begin{aligned}
& \delta(T(x), T(y)) \leq a \cdot d(x, y)+b \cdot \delta(x, T(x))+c \cdot \delta(y, T(y)) \\
& \text { for each }(x, y) \in \operatorname{Graf}(T) .
\end{aligned}
$$

Then $\operatorname{Fix}(T)=\operatorname{SFix}(T) \neq \emptyset$.

Proof. Let $q>1$ and $x_{0} \in X$ be arbitrary. Then there exists $x_{1} \in T\left(x_{0}\right)$ such that $\delta\left(x_{0}, T\left(x_{0}\right)\right) \leq q \cdot d\left(x_{0}, x_{1}\right)$. We have $\delta\left(x_{1}, T\left(x_{1}\right)\right) \leq \delta\left(T\left(x_{0}\right), T\left(x_{1}\right)\right) \leq a \cdot d\left(x_{0}, x_{1}\right)+$ $b \cdot \delta\left(x_{0}, T\left(x_{0}\right)\right)+c \cdot \delta\left(x_{1}, T\left(x_{1}\right)\right) \leq a d\left(x_{0}, x_{1}\right)+b q d\left(x_{0}, x_{1}\right)+c \cdot \delta\left(x_{1}, T\left(x_{1}\right)\right)$. Hence $\delta\left(x_{1}, T\left(x_{1}\right)\right) \leq \frac{a+b q}{1-c} \cdot d\left(x_{0}, x_{1}\right)$. By this procedure, we can obtain the sequence $\left(x_{n}\right)_{n \in \mathbb{N}}$ having the property $d\left(x_{n}, x_{n+1}\right) \leq\left(\frac{a+b q}{1-c}\right)^{n} \cdot d\left(x_{0}, x_{1}\right)$ for each $n \in \mathbb{N}$. If we choose $q>\frac{b}{1-a-c}$ then $\frac{a+b q}{1-c}<1$. Hence $\left(x_{n}\right)_{n \in \mathbb{N}}$ is a Cauchy sequence in the complete metric space $(X, d)$. Denote by $x^{*}$ the limit of the sequence $\left(x_{n}\right)_{n \in \mathbb{N}}$. Since $\operatorname{Graf}(T)$ is a closed set in $X \times X$ we obtain the first conclusion $x^{*} \in T\left(x^{*}\right)$.

Let us establish now the relation $\operatorname{Fix}(T)=\operatorname{SFix}(T)$. It is enough to prove that $\operatorname{Fix}(T) \subset \operatorname{SFix}(T)$. For, let $x \in \operatorname{Fix}(T)$ be arbitrary. Then, using the hypothesis (with $y=x \in T(x))$ we get successively: $\delta(T(x)) \leq(b+c) \cdot \delta(x, T(x)) \leq(b+c) \cdot \delta(T(x))$. Suppose, by absurdum, that card $T(x)>1$. Then $\delta(T(x))>0$ and using the above relation we get $1 \leq b+c$, a contradiction. Hence $\delta(T(x))=0$ and so $\{x\}=T(x)$.

REMARK 3.3. Theorem 3.2 is an extension of some results given in S. Reich [16] and I. A. Rus [21].

Moreover, condition (3.1) can be replaced with a more general one, namely: there exist $a \in[0,1[$ such that

$$
\delta(T(x), T(y)) \leq a \cdot \delta(x, T(x)) \text { for each }(x, y) \in \operatorname{Graf}(T),
$$

since $d(x, y) \leq \delta(x, T(x))$ and $\delta(y, T(y)) \leq \delta(T(x), T(y))$.

Next, we present a strict fixed point theorem.

TheOrem 3.4. Let $(X, d)$ be a complete metric space, and $T: X \rightarrow P_{\mathrm{b}}(X)$ be a set-valued operator. Suppose that

there exist $a, b \in \mathbb{R}_{+}$with $a+b<1$ such that for each $x \in X$

$$
\text { there exists } y \in T(x) \text { with } \delta(y, T(y)) \leq a \cdot d(x, y)+b \cdot \delta(x, T(x)) \text {. }
$$

If the map $f: X \rightarrow \mathbb{R}_{+}$, defined by $f(x):=\delta(x, T(x))$, is lower semicontinuous, then $\operatorname{SFix}(T) \neq \emptyset$. 
Proof. From (3.2), for each $x \in X$ there is a $y \in T(x)$ such that $\delta(y, T(y)) \leq(a+b) \times$ $\delta(x, T(x))$. Then, for each $x_{0} \in X$ we can construct inductively a sequence $\left(x_{n}\right)_{n \in \mathbb{N}}$ of successive approximations for $T$ starting from $x_{0}$, having the property $\delta\left(x_{n}, T\left(x_{n}\right)\right) \leq$ $(a+b)^{n} \cdot \delta\left(x_{0}, T\left(x_{0}\right)\right)$. Hence, we will obtain $d\left(x_{n}, x_{n+1}\right) \leq \delta\left(x_{n}, T\left(x_{n}\right)\right) \rightarrow 0$ as $n \rightarrow+\infty$. As a consequence, the sequence $\left(x_{n}\right)_{n \in \mathbb{N}}$ is Cauchy. Denote by $x^{*} \in X$ the limit of this sequence.

If we denote $f\left(x_{n}\right):=\delta\left(x_{n}, T\left(x_{n}\right)\right)$, then using the lower semicontinuity of $f$ we can write:

$$
0 \leq f\left(x^{*}\right) \leq \liminf _{n \rightarrow+\infty} f\left(x_{n}\right)=0 .
$$

So, $f\left(x^{*}\right)=0$ and the conclusion $\left\{x^{*}\right\}=T\left(x^{*}\right)$ follows.

REMARK 3.5. If, instead of the lower semicontinuity of $f$, we suppose that $\operatorname{Graf}(T)$ is closed, then, since $\left(x_{n}\right)_{n \in \mathbb{N}}$ is a sequence of successive approximations for $T$, we immediately get that $x^{*} \in T\left(x^{*}\right)$. So, the conclusion of the above result is $\operatorname{Fix}(T) \neq \emptyset$. It is an open question if the above fixed point is a strict fixed point for $T$.

REMARK 3.6. In Theorem 3.4 condition (3.2) can be replaced with a more general one: there exists $a \in[0,1$ [ such that for each $x \in X$ there exists $y \in T(x)$ with $\delta(y, T(y)) \leq$ $a \cdot \delta(x, T(x))$, since again $d(x, y) \leq \delta(x, T(x))$.

REMARK 3.7. An open problem is to construct a fixed point theory for set-valued $Y$-contractions in $K$-metric spaces (see [27]).

4. Set-valued contractions outside a bounded set. Let $X$ be a Banach space and $T: X \rightarrow P_{b}(X)$. By definition (see [10], [7]), the operator $T$ is called quasi-bounded if there exist $m, M>0$ such that

$$
\|y\| \leq m \cdot\|x\|+M \text { for each }(x, y) \in \operatorname{Graf}(T) .
$$

The number

$$
|T|:=\inf \{m>0: \text { there exists } M>0 \text { such that relation (4.1) holds }\},
$$

is called the quasi-norm of $T$.

Let us denote by $\alpha_{K}$ the Kuratowski measure of noncompactness on $X$ and let $\varphi: \mathbb{R}_{+} \rightarrow \mathbb{R}_{+}$be a comparison function (i.e. $\varphi$ is non-decreasing and $\left(\varphi^{n}(t)\right)_{n \in \mathbb{N}}$ goes to 0 , for each $t \geq 0$ as $n \rightarrow+\infty)$. An operator $T: X \rightarrow P_{b}(X)$ is said to be an $\left(\alpha_{K}, \varphi\right)$ contraction if $T$ is a bounded operator, $\varphi$ is a comparison function and $\alpha_{K}(T(A)) \leq$ $\varphi\left(\alpha_{K}(A)\right)$ for each $A \in P_{b}(X) \cap I(T)$.

The first main result of this section is

Theorem 4.1. Let $X$ be a Banach space, $Z \in P_{b}(X)$ and $T: X \rightarrow P_{\mathrm{cp}, \mathrm{cv}}(X)$. Suppose that the following assertions hold:

(i) $T$ is u.s.c. and compact (i.e. $T$ sends bounded sets into relatively compact sets);

(ii) there exists $a \in] 0,1[$ such that

$$
H\left(T\left(x_{1}\right), T\left(x_{2}\right)\right) \leq a \cdot\left\|x_{1}-x_{2}\right\| \text { for each }\left(x_{1}, x_{2}\right) \in(X \backslash Z) \times(X \backslash Z) .
$$

Then $\operatorname{Fix}(T) \neq \emptyset$. 
Proof. From (ii) the operator $T$ is quasi-bounded with the quasi-norm $|T|=a<1$. For, consider first $x \in Z$. Then $\|T(x)\|:=\sup _{y \in T(x)}\|y\| \leq\|T(Z)\|<+\infty$. If $x \in X \backslash Z$ then consider an arbitrary but fixed $x_{0} \in X \backslash Z$. We have $\|T(x)\|=H(T(x),\{0\}) \leq$ $H\left(T(x), T\left(x_{0}\right)\right)+H\left(T\left(x_{0}\right),\{0\}\right) \leq a \cdot\left\|x-x_{0}\right\|+\left\|T\left(x_{0}\right)\right\| \leq a \cdot\|x\|+a \cdot\left\|x_{0}\right\|+\left\|T\left(x_{0}\right)\right\|$. Hence, for all $x \in X$ we get $\|T(x)\| \leq a \cdot\|x\|+\max \left(a \cdot\left\|x_{0}\right\|+\left\|T\left(x_{0}\right)\right\|,\|T(Z)\|\right)$. From Lemma 2.1 in [10] (p. 59) there exists $R>0$ such that $T(\widetilde{B}(0, R)) \subset \widetilde{B}(0, R))$. If we take into account that $T(\widetilde{B}(0, R))$ is an invariant subset for $T$, the proof follows now from the Bohnenblust-Karlin fixed point theorem (see [20] and the references therein).

A more general result of this type is

Theorem 4.2. Let $X$ be a Banach space, $Z \in P_{b}(X)$ and $T: X \rightarrow P_{\mathrm{cp}, \mathrm{cv}}(X)$. Suppose that the following assertions hold:

(i) $T$ is an u.s.c. $\left(\alpha_{K}, \varphi\right)$-contraction;

(ii) there exists $a \in] 0,1[$ such that

$$
H\left(T\left(x_{1}\right), T\left(x_{2}\right)\right) \leq a \cdot\left\|x_{1}-x_{2}\right\| \text { for each }\left(x_{1}, x_{2}\right) \in(X \backslash Z) \times(X \backslash Z) .
$$

Then $\operatorname{Fix}(T) \neq \emptyset$.

Proof. The proof runs in a similar way to the above one, with the only difference that, instead of the Bohnenblust-Karlin fixed point theorem, we use Theorem 4.7 in [20].

In terms of the fixed point structures we can prove the following abstract result.

Theorem 4.3. Let $\left(X, S(X), M^{0}\right)$ be a f.p.s. on a Banach space $X, T: X \multimap X$ and $Z \in P_{b}(X)$. We suppose that

(i) $\widetilde{B}(0, R)) \in S(X)$ for each $R>0$;

(ii) $T \in M^{0}(Y)$ for each $Y \in S(X)$;

(iii) there exists $a \in] 0,1[$ such that

$$
H\left(T\left(x_{1}\right), T\left(x_{2}\right)\right) \leq a \cdot\left\|x_{1}-x_{2}\right\| \text { for each }\left(x_{1}, x_{2}\right) \in(X \backslash Z) \times(X \backslash Z) .
$$

Then $\operatorname{Fix}(T) \neq \emptyset$.

The above considerations give rise to the following open question:

Problem 4.4. Which generalized set-valued contractions $T: X \rightarrow P_{\mathrm{b}, \mathrm{cl}, \mathrm{cv}}(X)$ are quasibounded with the quasi-norm $|T|<1$ ?

For the single-valued case see [24] (p. 20) and the references therein (M. C. Anisiu (1983), F. Aldea (2002)).

\section{Cyclic representation of an invariant subset with respect to a set-valued} operator. Let us observe first that the results in Kirk, Srinivasan, Veeramani [9] and I. A. Rus [23] give rise to the following concept:

Definition 5.1. Let $X$ be a nonempty set and $T: X \rightarrow P(X)$ a set-valued operator. By definition, $X=\bigcup_{i=1}^{m} X_{i}$ (where $X_{i} \subset X$ for each $i \in\{1,2, \ldots, m\}$ ) is a cyclic representation of $X$ with respect to $T$ if $T\left(X_{1}\right) \subset X_{2}, \ldots, T\left(X_{m-1}\right) \subset X_{m}, T\left(X_{m}\right) \subset X_{1}$. 
EXAmPLE 5.2. Let $X$ be a nonempty set and $T: X \rightarrow P(X)$ a set-valued operator. We suppose that there exist $A \subset X$ and $n_{0} \in \mathbb{N}^{*}$ such that $T^{n_{0}}(A) \subset A$. Then $B:=$ $\bigcup_{i=1}^{n_{0}-1} T^{i}(A)$ is a cyclic representation of $B$ with respect to $T$. Moreover $B \in I(T)$.

REMARK 5.3. If $X:=\bigcup_{i=1}^{m} X_{i}$ is a cyclic representation of $X$ with respect to $T: X \rightarrow$ $P(X)$, then $X_{i}(i \in\{1,2, \ldots, m\})$ are invariant subsets for $T^{m}$.

The following abstract result is important in our considerations.

Theorem 5.4. Let $(X, \rightarrow)$ be an L-space, $T: X \rightarrow P(X)$ a set-valued operator and $X:=\bigcup_{i=1}^{m} X_{i}$ be a cyclic representation of $X$ with respect to $T$. Suppose that

(i) $X_{i} \in P_{\mathrm{cl}}(X)$ for each $i \in\{1,2, \ldots, m\}$;

(ii) there exists a convergent sequence $\left(x_{n}\right)_{n \in \mathbb{N}}$, where $x_{n} \in X, x_{n+1} \in T\left(x_{n}\right)$ for each $n \in \mathbb{N}$.

Then $\bigcap_{i=1}^{m} X_{i} \neq \emptyset$.

Proof. Denote by $x^{*}$ the limit of the sequence $\left(x_{n}\right)_{n \in \mathbb{N}}$. Because $x_{n} \in \bigcup_{i=1}^{m} X_{i}$ for each $n \in \mathbb{N}$, it follows that infinitely many elements of this sequence lie in each $X_{i}$, $i \in\{1,2, \ldots, m\}$. From (i) one sees that $x^{*} \in X_{i}, i \in\{1,2, \ldots, m\}$. Hence $\bigcap_{i=1}^{m} X_{i} \neq \emptyset$.

As consequence of the above result we have

TheOREM 5.5. Let $\left(X, S(X), M^{0}\right)$ be a fixed point structure, where $(X, \rightarrow)$ is an L-space. Let $A_{i} \in P_{\mathrm{cl}}(X)$ for each $i \in\{1,2, \ldots, m\}$. Define $Y:=\bigcup_{i=1}^{m} A_{i}$ and consider $T: Y \rightarrow$ $P(Y)$. Suppose that

(i) $Y:=\bigcup_{i=1}^{m} A_{i}$ is a cyclic representation of $Y$ with respect to $T$;

(ii) there exists a convergent sequence $\left(x_{n}\right)_{n \in \mathbb{N}}$, where $x_{n} \in X, x_{n+1} \in T\left(x_{n}\right)$ for each $n \in \mathbb{N}$;

(iii) if $A:=\bigcap_{i=1}^{m} A_{i} \neq \emptyset$ then $A \in S(X)$ and $T_{\left.\right|_{A}} \in M(A)$.

Then $\operatorname{Fix}(T) \neq \emptyset$.

Proof. From (i), (ii) and Theorem 5.4 it follows that $A \neq \emptyset$. From the definition of the fixed point structure and using the assertion (iii) we have $\operatorname{Fix}(T) \neq \emptyset$.

THEOREM 5.6. Let $\left(X, S(X), M^{0}\right)$ be a fixed point structure, where $X$ is a nonempty set. Let $A_{i} \in P(X)$ for each $i \in\{1,2, \ldots, m\}$. Define $Y:=\bigcup_{i=1}^{m} A_{i}$ and consider $T: Y \rightarrow$ $P(Y)$. Suppose that

(i) $\left(A_{i}\right)_{i \in\{1,2, \ldots, m\}}$ is a cyclic representation of $Y$ with respect to $T$;

(ii) $A_{i} \in S(X)$ for some $i \in\{1,2, \ldots, m\}$;

(iii) $G_{1}, G_{2} \in M^{0}$ implies $G_{1} \circ G_{2} \in M^{0}$. 
Then $\operatorname{Fix}\left(T^{m}\right) \neq \emptyset$.

Proof. Let, for example, $A_{i} \in S(X)$. From Definition 5.1 and (iii) it follows that $T^{m} \in M^{0}\left(A_{i}\right)$. The proof follows now from Remark 5.3.

Some applications of the above results can be obtained by taking in Theorems 5.5 and 5.6 some particular fixed point structures (see I. A. Rus [20]).

\section{References}

[1] J. Andres, J. Fišer, Metric and topological multivalued fractals, Internat. J. Bifur. Chaos Appl. Sci. Engrg. 14 (2004), 1277-1289.

[2] J. Andres, L. Górniewicz, On the Banach contraction principle for multivalued mappings, in: Approximation, Optimization and Mathematical Economics (Pointe-à-Pitre 1999), Physica, Heidelberg, 2001, 1-23.

[3] H. Covitz, S. B. Nadler, Jr., Multi-valued contraction mappings in generalized metric spaces, Israel J. Math. 8 (1970), 5-11.

[4] M. Fréchet, Les espaces abstraits, Gauthier-Villars, Paris 1928.

[5] M. Frigon, A. Granas, Résultats du type de Leray-Schauder pour les contractions multivoques, Topol. Methods Nonlinear Anal. 4 (1994), 197-208.

[6] A. Granas, J. Dugundji, Fixed Point Theory, Springer Monogr. Math., Springer, New York 2003.

[7] R. Iannaci, The spectrum for nonlinear multi-valued maps via approximations, Boll. Un. Mat. Ital. B (5) 15 (1978), 527-545.

[8] W. A. Kirk, B. Sims (eds.), Handbook of Metric Fixed Point Theory, Kluwer Acad. Publ., Dordrecht 2001.

[9] W. A. Kirk, P. S. Srinivasan, P. Veeramani, Fixed points for mappings satisfying cyclical contractive conditions, Fixed Point Theory 4 (2003), 79-89.

[10] M. Martelli, A. Vignoli, Some surjectivity results for non-compact multi-valued maps, Rend. Accad. Sci. Fis. Mat. Napoli (4) 41 (1974), 57-66.

[11] S. B. Nadler, Jr., Multivalued contraction mappings, Pacific J. Math. 30 (1969), 475-488.

[12] A. Petruşel, On Frigon-Granas type multifunctions, Nonlinear Anal. Forum 7 (2002), 113-121.

[13] A. Petruşel, Multivalued weakly Picard operators and applications, Sci. Math. Jpn. 59 (2004), 169-202.

[14] A. Petruşel, I. A. Rus, Fixed point theorems in ordered L-spaces, Proc. Amer. Math. Soc. 134 (2006), 411-418.

[15] A. C. M. Ran, M. C. B. Reurings, A fixed point theorem in partially ordered sets and some applications to matrix equations, Proc. Amer. Math. Soc. 132 (2004), 1435-1443.

[16] S. Reich, Fixed points of contractive functions, Boll. Un. Mat. Ital. (4) 5 (1972), 26-42.

[17] I. A. Rus, The method of successive approximations, Rev. Roumaine Math. Pures Appl. 17 (1972), 1433-1437 (in Russian).

[18] I. A. Rus, Fixed point theorems for multivalued mappings in complete metric spaces, Math. Japon. 20 (1975), special issue, 21-24.

[19] I. A. Rus, Normcontraction mappings outside a bounded subset, Itinerant Sem. on Functional Equations, Approx. and Convexity, 1986, 257-260. 
[20] I. A. Rus, Technique of the fixed point structures for multivalued mappings, Math. Japon. 38 (1993), 289-296.

[21] I. A. Rus, Generalized Contractions and Applications, Cluj Univ. Press, Cluj-Napoca 2001.

[22] I. A. Rus, Strict fixed point theory, Fixed Point Theory 4 (2003), 177-183.

[23] I. A. Rus, Cyclic representations and fixed points, Annals of the Tiberiu Popoviciu Seminar, 2005, to appear.

[24] I. A. Rus, A. Petruşel, G. Petruşel, Fixed Point Theory 1950-2000: Romanian Contributions, House of the Book of Science, Cluj-Napoca 2002.

[25] I. A. Rus, A. Petruşel, A. Sîntămărian, Data dependence of the fixed point set of some multivalued weakly Picard operators, Nonlinear Anal. 52 (2003), 1947-1959.

[26] J. Saint-Raymond, Multivalued contractions, Set-Valued Anal. 2 (1994), 559-571.

[27] P. P. Zabreiko, K-metric and K-normed linear spaces: survey, Collect. Math. 48 (1997), 825-859. 
